PROCEEDINGS OF THE

AMERICAN MATHEMATICAL SOCIETY

Volume 140, Number 4, April 2012, Pages 1403-1411

S 0002-9939(2011)11143-2

Article electronically published on August 10, 2011

\title{
THE FULL GROUP C*-ALGEBRA OF THE MODULAR GROUP IS PRIMITIVE
}

\author{
ERIK BÉDOS AND TRON Å. OMLAND
}

(Communicated by Marius Junge)

This paper is dedicated to the memory of Gerard J. Murphy

\begin{abstract}
We show that the full group $\mathrm{C}^{*}$-algebra of $\operatorname{PSL}(n, \mathbb{Z})$ is primitive when $n=2$ and not primitive when $n \geq 3$. Moreover, we show that there exists an uncountable family of pairwise inequivalent, faithful irreducible representations of $C^{*}(P S L(2, \mathbb{Z}))$.
\end{abstract}

\section{INTRODUCTION}

Simple and, more generally, primitive and prime $\mathrm{C}^{*}$-algebras may be considered as building blocks of the theory, playing a somewhat similar role as factors do within the theory of von Neumann algebras. If we restrict ourselves to separable $\mathrm{C}^{*}$-algebras, as we always do in this paper, primitivity is equivalent to primeness (see for example [17]), and we will therefore refer to primitivity for both notions. Now, given some class of separable $\mathrm{C}^{*}$-algebras, one natural task is to investigate which members of this class are simple or primitive.

An interesting family of separable $\mathrm{C}^{*}$-algebras consists of the group $\mathrm{C}^{*}$-algebras associated with countable discrete groups. We recall that such a group $G$ is called $C^{*}$-simple if its reduced group $\mathrm{C}^{*}$-algebra $C_{r}^{*}(G)$ is simple. As the full group $\mathrm{C}^{*}$ algebra $C^{*}(G)$ is simple only when $G$ is trivial, this terminology is not ambiguous. The class of $\mathrm{C}^{*}$-simple groups has received a lot of attention during the last decades and the reader may consult 8 for a recent, comprehensive review. It is also well known (see [15, 14]) that $C_{r}^{*}(G)$ is primitive if and only if $G$ is icc (that is, every nontrivial conjugacy class in $G$ is infinite) if and only if the group von Neumann algebra of $G$ is a factor.

On the other hand, the problem of determining when $C^{*}(G)$ is primitive seems hard in general. A necessary condition is that $G$ is icc [14, and this condition is also sufficient when $G$ is assumed to be amenable, as $C^{*}(G)$ is then isomorphic to $C_{r}^{*}(G)$. We note in passing that this problem is quite different from the one of determining the class of groups having a faithful irreducible unitary representation, which contains many other groups besides all icc groups (see [3]).

Until a few years ago, the only known nonamenable icc groups having a primitive full group $\mathrm{C}^{*}$-algebra were nonabelian free groups, as originally shown by

Received by the editors January 6, 2010, and in revised form, January 7, 2011.

2010 Mathematics Subject Classification. Primary 46L05; Secondary 22D25, 46L55.

Both authors are partially supported by the Norwegian Research Council (NFR).

(C)2011 American Mathematical Society

Reverts to public domain 28 years from publication 
H. Yoshizawa 21] and rediscovered later by M. D. Choi [5, 6]. Then primitivity of $C^{*}(G)$ was established when $G=G_{1} * G_{2}$ is the free product of two countable subgroups $G_{1}$ and $G_{2}$ satisfying at least one of the following assumptions:

i) $G_{1}=\mathbb{Z} * \mathbb{Z}$ or $G_{1}=\mathbb{Z} * \mathbb{Z}_{2} \quad\left(G_{2}\right.$ being then any group $)$.

ii) $G_{1}$ is nontrivial and free, and $G_{2}$ is nontrivial and amenable.

iii) $G_{1}$ is nonabelian and free, and $C^{*}\left(G_{2}\right)$ admits no nontrivial projections.

Case (i) is due to N. Khattou [9, Théorèmes 2 and 3], while (ii) and (iii) are due to G. J. Murphy [14, Theorems 3.3 and 3.4].

In [8, Problem 25], P. de la Harpe raises the problem of finding other (nonamenable icc) groups having a primitive full group $\mathrm{C}^{*}$-algebra. One may especially wonder whether this property holds for any group $G$ which is the free product of two nontrivial groups, where at least one of them has more than two elements (as the infinite dihedral group $\mathbb{Z}_{2} * \mathbb{Z}_{2}$ is not icc). The simplest case for which the answer is unknown is the modular group $\operatorname{PSL}(2, \mathbb{Z})=\mathbb{Z}_{2} * \mathbb{Z}_{3}$, and our main result in this paper is that $C^{*}(P S L(2, \mathbb{Z})$ ) is indeed primitive (cf. Theorem 2.3).

An outline of our proof is as follows. Let $H$ be the kernel of the canonical homomorphism from $G=\mathbb{Z}_{2} * \mathbb{Z}_{3}$ onto $\mathbb{Z}_{2} \times \mathbb{Z}_{3}$. Then $H \simeq \mathbb{Z} * \mathbb{Z}$. Exploiting a certain phase-action of the circle group $\mathbb{T}$ on $C^{*}(H)$, we then show how a faithful irreducible representation of $C^{*}(H)$ may be picked so that it induces a representation of $C^{*}(G)$ which is also faithful and irreducible. Moreover, we show that there exists an uncountable family of pairwise inequivalent, irreducible faithful representations of $C^{*}(G)$. A similar idea was used by Murphy in his proof of [14, Theorem 3.3], where he considers certain semidirect products of nonabelian free groups by amenable groups. However, in our case, the exact sequence $1 \rightarrow H \rightarrow G \rightarrow \mathbb{Z}_{2} \times \mathbb{Z}_{3} \rightarrow 1$ does not split, so we have to decompose $C^{*}(G)$ as a twisted crossed product of $C^{*}(H)$ by $\mathbb{Z}_{2} \times \mathbb{Z}_{3}$ and use results of J. Packer and I. Raeburn from [16. Actually, when $H$ is a normal subgroup of a group $G$, we give a criterion ensuring that primitivity of $C^{*}(H)$ passes over to $C^{*}(G)$ (see Theorem 2.11) and use it to deduce Theorem 2.31

Murphy mentions in 14 that he knows of no example of an icc group whose full group $\mathrm{C}^{*}$-algebra is not primitive, but that it is unlikely that such groups do not exist. Now it is almost immediate (cf. Proposition 2.5) that $C^{*}(G)$ is not primitive whenever $G$ is a nontrivial group having Kazhdan's property (T). As there are many nontrivial icc groups having property $(\mathrm{T})$, such as $G=P S L(n, \mathbb{Z})$ for any integer $n \geq 3$ (see [4), this confirms that the full group $\mathrm{C}^{*}$-algebra of an icc group is not necessarily primitive. Moreover, as it is known that $P S L(n, \mathbb{Z})$ is always $\mathrm{C}^{*}$-simple (see [1, 2]), this also illustrates that $\mathrm{C}^{*}$-simplicity of $G$ does not imply that $C^{*}(G)$ is primitive.

\section{ON PRIMITIVITY OF FULL GROUP $\mathrm{C}^{*}$-ALGEBRAS AND THE MODULAR GROUP}

We use standard notation and terminology in operator algebras; see for example 17. 17, 6]. All Hilbert spaces are assumed to be complex. By a representation of a $\mathrm{C}^{*}$-algebra $A$, we always mean a $*$-homomorphism $\pi: A \rightarrow \mathcal{B}(\mathcal{H})$ into the bounded operators $\mathcal{B}(\mathcal{H})$ on some Hilbert space $\mathcal{H}$. We use the same symbol $\simeq$ to

\footnotetext{
${ }^{1}$ In a recent paper (Banach J. of Math. Anal. 5 (2011), 44-58), we use this criterion to show that $C^{*}(G)$ is primitive whenever $G$ is the free product of two nontrivial amenable groups where at least one of them has more than two elements. The proof is combinatorially much more involved than in the case of the modular group.
} 
denote unitary equivalence of operators on Hilbert spaces, (unitary) equivalence of representations of a $\mathrm{C}^{*}$-algebra and $*$-isomorphism between $\mathrm{C}^{*}$-algebras.

All the groups we consider are assumed to be countable and discrete. If $G$ is such a group, we let $e_{G}$, or just $e$, denote its unit. When $G$ acts on a nonempty set $X$ and $x \in X$, we say that $x$ is a free point (for the action of $G$ ) whenever $g \cdot x \neq x$ for all $g \in G, g \neq e$.

Let $A$ be a separable $\mathrm{C}^{*}$-algebra and let $\widehat{A}$ denote the set of (unitary) equivalence classes of nonzero irreducible representations of $A$. Set

$$
\widehat{A}^{o}=\{[\pi] \in \widehat{A} \mid \pi \text { is faithful }\} .
$$

This set is clearly well-defined, and it is nonempty if and only if $A$ is primitive.

Assume now that a group $G$ has a normal subgroup $H$ such that $A=C^{*}(H)$ is primitive and set $K=G / H$. Then $K$ acts on $\widehat{A}^{\circ}$ in a natural way.

To see this, let $n: K \rightarrow G$ be a normalized section for the canonical homomorphism $p$ from $G$ onto $K$ (so $n\left(e_{K}\right)=e_{G}$ and $p \circ n$ gives the identity map on $K)$.

Let $\alpha: K \rightarrow \operatorname{Aut}(A)$ and $u: K \times K \rightarrow A$ be determined by

$$
\begin{gathered}
\alpha_{k}\left(i_{H}(h)\right)=i_{H}\left(n(k) h n(k)^{-1}\right), \quad k \in K, h \in H, \\
u(k, l)=i_{H}\left(n(k) n(l) n(k l)^{-1}\right), \quad k, l \in K,
\end{gathered}
$$

where $i_{H}$ denotes the canonical injection of $H$ into $A$. Then $(\alpha, u)$ is a twisted action of $K$ on $A$ (see [16] or the Appendix); specifically, we have

$$
\alpha_{k} \alpha_{l}=\operatorname{Ad}(u(k, l)) \alpha_{k l}, k, l \in K,
$$

where, as usual, $\operatorname{Ad}(v)$ denotes the inner automorphism implemented by some unitary $v$ in $A$.

This twisted action $(\alpha, u)$ clearly induces an action of $K$ on $\widehat{A}$ given by

$$
k \cdot[\pi]=\left[\pi \circ \alpha_{k^{-1}}\right] .
$$

By restriction, we get the natural action of $K$ on $\widehat{A}^{o}$, which is easily seen to be independent of the choice of normalized section $n$ for $p$.

The following result holds.

Theorem 2.1. Assume that a group $G$ has a normal subgroup $H$ such that

(a) $A=C^{*}(H)$ is primitive,

(b) $K=G / H$ is amenable,

(c) the natural action of $K$ on $\widehat{A}^{o}$ has a free point.

Then $C^{*}(G)$ is primitive.

Proof. We use the notation introduced above and recall that Packer and Raeburn have shown (see [16. Theorem 4.1]) that $C^{*}(G)$ may be decomposed as the twisted crossed product associated with $(\alpha, u)$ :

$$
C^{*}(G) \simeq A \times_{\alpha, u} K .
$$

Let $[\pi] \in \widehat{A}^{o}$ be a free point for the natural action of $K$. This means that

$$
\pi \circ \alpha_{k} \neq \pi \text { for all } k \in K, k \neq e .
$$

Now, this condition implies that the induced regular representation Ind $\pi$ of $A \times{ }_{\alpha, u} K$ is irreducible. Indeed, as $G$ is discrete, this could be deduced from 11] (see the 
discussion in [18, Introduction]; see also [12, 13, 19]). For completeness, we give a proof in the Appendix (cf. Corollary 3.2(a)).

Further, as $K$ is amenable, [16, Theorem 3.1] gives that Ind $\pi$ is faithful. Altogether, it follows that $C^{*}(G)$ has a faithful irreducible representation, as desired.

Remark 2.2. Assume that $G$ has a normal subgroup $H$ and that $K=G / H$. It would be interesting to find more general conditions than those given in Theorem 2.1 ensuring that $C^{*}(G)$ is primitive. However, even for the case where $G$ is the direct product of $H$ and $K$, this is a nontrivial problem. Murphy has shown in [14. Theorem 2.5] that $C^{*}(H \times K)$ is primitive whenever $C^{*}(H)$ is primitive and $K$ is amenable and icc. But when for example $\mathbb{F}$ is a free nonabelian group, it is unknown whether $C^{*}(\mathbb{F} \times \mathbb{F})$ is primitive or not. Note that if it should happen that $C^{*}(\mathbb{F} \times \mathbb{F})$ is not primitive, this would imply that

$$
C^{*}(\mathbb{F}) \otimes_{\max } C^{*}(\mathbb{F}) \not C^{*}(\mathbb{F}) \otimes_{\min } C^{*}(\mathbb{F}) .
$$

Thus, when $\mathbb{F}$ has infinitely many generators, this would solve negatively an open problem of E. Kirchberg, which is known to be equivalent to Connes' famous embedding problem (see [10]).

Theorem 2.3. Set $G=P S L(2, \mathbb{Z})$. Then $C^{*}(G)$ is primitive. Moreover, there exists an uncountable family of pairwise inequivalent, irreducible faithful representations of $C^{*}(G)$.

Proof. Write $G=\mathbb{Z}_{2} * \mathbb{Z}_{3}=\left\langle a, b \mid a^{2}=b^{3}=1\right\rangle$ and let $H$ denote the kernel of the canonical homomorphism $p$ from $G$ onto $K=\mathbb{Z}_{2} \times \mathbb{Z}_{3}\left(\simeq \mathbb{Z}_{6}\right)$.

Then $H$ is freely generated as a group by $x_{1}=a b a b^{2}$ and $x_{2}=a b^{2} a b$ (see e.g. [20, I.1.3, Proposition 4]).

Set $A=C^{*}(H)$. Using [21] or [5], we may pick $[\pi] \in \widehat{A}^{o}$. Set

$$
U_{1}=i_{H}\left(x_{1}\right), V_{1}=\pi\left(U_{1}\right), \quad U_{2}=i_{H}\left(x_{2}\right), V_{2}=\pi\left(U_{2}\right),
$$

so $V_{1}, V_{2}$ are unitary operators on the separable Hilbert space $\mathcal{H}_{\pi}$ on which $\pi$ acts. As shown in the proof [5, Theorem 6], we may and do assume that $V_{2}$ is diagonal relative to some orthonormal basis for $\mathcal{H}_{\pi}$, with (distinct) diagonal entries given by some $\mu_{j} \in \mathbb{T}, j \in \mathbb{N}$.

For each $\lambda \in \mathbb{T}$, let $\gamma_{\lambda}$ be the $*$-automorphism of $A$ determined by

$$
\gamma_{\lambda}\left(U_{1}\right)=U_{1}, \gamma_{\lambda}\left(U_{2}\right)=\lambda U_{2},
$$

and set $\pi_{\lambda}=\pi \circ \gamma_{\lambda}$. Clearly, $\left[\pi_{\lambda}\right] \in \widehat{A}^{o}$.

We will show that we can pick $\lambda \in \mathbb{T}$ such that $\left[\pi_{\lambda}\right]$ is a free point for the natural action of $K$ on $\widehat{A}^{o}$. As $K$ is amenable, the primitivity of $C^{*}(G)$ will then follow from Theorem 2.1. To pick $\lambda$, we proceed as follows.

As a normalized section for $p: G \rightarrow K$, we choose $n: K \rightarrow G$ given by

$$
n(i, j)=a^{i} b^{j}, \quad i \in\{0,1\}, j \in\{0,1,2\} .
$$

For each $k=(i, j) \in K$ we let $\alpha_{k}$ be the $*$-automorphism of $A$ used to define the natural action of $K$ on $\widehat{A}^{o}$.

It is clear that $\left[\pi_{\lambda}\right]$ will be a free point for this action of $K$ if for each $k \in K$, $k \neq(0,0)$, we have

$$
\left(\pi_{\lambda} \circ \alpha_{k}\right)\left(U_{r}\right) \not \pi_{\lambda}\left(U_{r}\right) \text { for } r=1 \text { or } r=2 .
$$


Some elementary computations give:

$$
\pi_{\lambda}\left(U_{1}\right)=V_{1}, \pi_{\lambda}\left(U_{2}\right)=\lambda V_{2}
$$

when $k=(0,1): \quad\left(\pi_{\lambda} \circ \alpha_{k}\right)\left(U_{2}\right)=V_{1}^{*}$;

when $k=(0,2): \quad\left(\pi_{\lambda} \circ \alpha_{k}\right)\left(U_{1}\right)=\left(\lambda V_{2}\right)^{*}$;

when $k=(1,0): \quad\left(\pi_{\lambda} \circ \alpha_{k}\right)\left(U_{2}\right)=\left(\lambda V_{2}\right)^{*}$;

when $k=(1,1): \quad\left(\pi_{\lambda} \circ \alpha_{k}\right)\left(U_{2}\right)=V_{1}$

when $k=(1,2): \quad\left(\pi_{\lambda} \circ \alpha_{k}\right)\left(U_{1}\right)=\lambda V_{2}$.

It follows that $\left[\pi_{\lambda}\right]$ will be a free point whenever

$$
V_{1} \nsucceq \lambda V_{2}, V_{1} \nsucceq\left(\lambda V_{2}\right)^{*}, \lambda V_{2} \nsucceq\left(\lambda V_{2}\right)^{*} .
$$

Define

$$
\begin{gathered}
\Omega_{1}=\left\{\lambda \in \mathbb{T} \mid V_{1} \simeq \lambda V_{2}\right\}, \\
\Omega_{2}=\left\{\lambda \in \mathbb{T} \mid V_{1} \simeq\left(\lambda V_{2}\right)^{*}\right\},
\end{gathered}
$$

and

$$
\Omega_{3}=\left\{\lambda \in \mathbb{T} \mid \lambda V_{2} \simeq\left(\lambda V_{2}\right)^{*}\right\} .
$$

As the point spectrum of $V_{2}$ is given by $\sigma_{p}\left(V_{2}\right)=\left\{\mu_{j} \mid j \in \mathbb{N}\right\} \subseteq \mathbb{T}$, the sets $\Omega_{1}, \Omega_{2}$ and $\Omega_{3}$ are all countable.

Indeed, if $\Omega_{1}$ were uncountable, then, as $\sigma_{p}\left(V_{1}\right)=\lambda \sigma_{p}\left(V_{2}\right)$ for all $\lambda \in \Omega_{1}, \sigma_{p}\left(V_{1}\right)$ would also be uncountable; as $\mathcal{H}_{\pi}$ is separable, this is impossible. In the same way, we see that $\Omega_{2}$ must be countable. Finally, if $\Omega_{3}$ were uncountable, then the equality

$$
\lambda\left\{\mu_{j} \mid j \in \mathbb{N}\right\}=\bar{\lambda}\left\{\overline{\mu_{j}} \mid j \in \mathbb{N}\right\}
$$

would hold for uncountably many $\lambda$ 's in $\mathbb{T}$, and this is easily seen to be impossible.

Hence, the set $\Omega=\Omega_{1} \cup \Omega_{2} \cup \Omega_{3}$ is countable. Especially, $\Omega \neq \mathbb{T}$ and (*) holds for every $\lambda$ in the complement $\Omega^{c}$ of $\Omega$ in $\mathbb{T}$. Thus, we have shown that $C^{*}(G)$ is primitive.

To prove the second assertion, we consider $\lambda, \lambda^{\prime} \in \Omega^{c}$, so Ind $\pi_{\lambda}$ and Ind $\pi_{\lambda^{\prime}}$ are irreducible and faithful. A well-known argument (adapted to our twisted setting; see Corollary 3.2(b) in the Appendix) gives that Ind $\pi_{\lambda}$ and Ind $\pi_{\lambda^{\prime}}$ will be inequivalent whenever

$$
\pi_{\lambda} \circ \alpha_{j} \not \pi_{\lambda^{\prime}} \quad \text { for all } j \in K .
$$

Using our previous computations, we see that this will hold whenever

$$
\begin{gathered}
V_{1} \nsucceq \lambda V_{2}, V_{1} \nsucceq\left(\lambda V_{2}\right)^{*}, \\
V_{1} \nsucceq \lambda^{\prime} V_{2}, V_{1} \nsucceq\left(\lambda^{\prime} V_{2}\right)^{*}, \\
\lambda V_{2} \nsucceq \lambda^{\prime} V_{2},\left(\lambda V_{2}\right)^{*} \nsucceq \lambda^{\prime} V_{2} .
\end{gathered}
$$

The first four conditions are satisfied since $\lambda, \lambda^{\prime} \in \Omega^{c}$. Set

$$
\Omega_{\lambda}=\left\{\omega \in \mathbb{T} \mid \lambda V_{2} \simeq \omega V_{2} \text { or }\left(\lambda V_{2}\right)^{*} \simeq \omega V_{2}\right\} .
$$

Then $\Omega_{\lambda}$ is countable (arguing as in the first part of the proof), so $\Omega \cup \Omega_{\lambda}$ is countable. Hence, if we assume, as we may, that $\lambda^{\prime} \in\left(\Omega \cup \Omega_{\lambda}\right)^{c}$, then all six conditions above are satisfied, and it follows that Ind $\pi_{\lambda}$ and Ind $\pi_{\lambda^{\prime}}$ are inequivalent, irreducible and faithful.

Proceeding inductively, we may produce in this way a countably infinite family of pairwise inequivalent, irreducible faithful representations of $C^{*}(G)$. In fact, even an 
uncountable family of such representations does exist. Indeed, observe that Ind $\pi_{\lambda}$ is an essential representation of $C^{*}(G)$; that is, its range contains no compact operators other than zero. Otherwise, the irreducible representations Ind $\pi_{\lambda}$ and Ind $\pi_{\lambda^{\prime}}$ would have to be equivalent since they have the same kernel (cf. [7, Cor. 4.1.10]). As $C^{*}(G)$ is separable, the claim then follows from [7, Compléments 4.7.2].

Remark 2.4. Let $G=P S L(2, \mathbb{Z})$. As we have seen in the above proof, $C^{*}(G)$ has a faithful irreducible representation which is essential. Hence, $C^{*}(G)$ is antiliminary (cf. [7. Compléments 9.5.4]). Since $C^{*}(G)$ is also primitive (and therefore prime), it follows that the pure state space of $C^{*}(G)$ is weak* dense in the state space of $C^{*}(G)$ (cf. [7, Lemme 11.2.4]). This is also true when $G$ is a nonabelian free group; in fact, this is precisely what Yoshizawa proves in 21] when $G=\mathbb{F}_{2}$.

Our next observation is quite obvious and surely known to specialists.

Proposition 2.5. Let $G$ be a group with Kazhdan's property (T) (see e.g. [4) and assume that $C^{*}(G)$ is primitive. Then $G$ is trivial.

Proof. Set $A=C^{*}(G)$. We endow the primitive ideal space $\operatorname{Prim}(A)$ of $A$ with its Jacobson (hull-kernel) topology and $\widehat{A}$ with the weakest topology making the canonical map from $\widehat{A}$ onto $\operatorname{Prim}(A)$ continuous. Since $A$ is primitive, we may pick $\left[\pi_{0}\right] \in \widehat{A}^{o}$. As $\{0\}$ is dense in $\operatorname{Prim}(A),\left\{\left[\pi_{0}\right]\right\}$ is dense in $\widehat{A}$.

Now let $\pi_{1}$ denote the representation of $A$ associated with the trivial onedimensional unitary representation of $G$. Property $(\mathrm{T})$ means that $\left[\pi_{1}\right]$ is isolated in $\widehat{A}$; i.e. $\left\{\left[\pi_{1}\right]\right\}$ is open in $\widehat{A}$. Thus we must have $\left[\pi_{1}\right]=\left[\pi_{0}\right]$. Specifically, $\pi_{1}$ must be faithful, which implies that $G$ is trivial.

Corollary 2.6. Set $G=\operatorname{PSL}(n, \mathbb{Z}), n \geq 3$. Then $G$ is icc, but $C^{*}(G)$ is not primitive.

Proof. As it is well known that $G$ is icc and has property (T) (see 4]), this follows from Proposition 2.5.

Moreover, as $\operatorname{PSL}(n, \mathbb{Z})$ is always $\mathrm{C}^{*}$-simple (cf. [1, 2]), this result also shows that $\mathrm{C}^{*}$-simplicity of a group $G$ does not imply that $C^{*}(G)$ is primitive.

\section{Appendix}

We prove here a couple of results about induced representations of discrete twisted crossed products, which we could not find explicitly in the literature in the form needed for our purposes.

Let $(A, K, \alpha, u)$ be a twisted $C^{*}$-dynamical system as considered by Packer and Raeburn [16, where $A$ is a unital $C^{*}$-algebra, $K$ is a discrete group with unit $e$ and $(\alpha, u)$ is a twisted action of $K$ on $A$; this means that $\alpha$ is a map from $K$ into $\operatorname{Aut}(A)$, the group of $*$-automorphisms of $A$, and $u$ is a map from $K \times K \operatorname{into} \mathcal{U}(A)$, the unitary group of $A$, satisfying

$$
\begin{aligned}
\alpha_{k} \alpha_{l} & =\operatorname{Ad}(u(k, l)) \alpha_{k l}, \\
u(k, l) u(k l, m) & =\alpha_{k}(u(l, m)) u(k, l m), \\
u(k, e) & =u(e, k)=1
\end{aligned}
$$


for all $k, l, m \in K$. (To avoid technicalities, we assume that $A$ is unital; otherwise, one has to assume that the 2-cocycle $u$ takes its values in the unitary group of the multiplier algebra of $A$.)

The full twisted crossed product $A \times_{\alpha, u} K$ may then be considered as the enveloping $\mathrm{C}^{*}$-algebra of the Banach *-algebra $\ell^{1}(A, K, \alpha, u)$, which consists of the Banach space $\ell^{1}(K, A)$ equipped with product and involution given by

$$
\begin{gathered}
(f * g)(l)=\sum_{k \in K} f(k) \alpha_{k}\left(g\left(k^{-1} l\right)\right) u\left(k, k^{-1} l\right), \quad f, g \in \ell^{1}(K, A), l \in K, \\
f^{*}(l)=u\left(l, l^{-1}\right)^{*} \alpha_{l}\left(f\left(l^{-1}\right)\right)^{*}, \quad f \in \ell^{1}(K, A), l \in K .
\end{gathered}
$$

We let $i_{K}$ and $i_{A}$ denote the canonical injections of $K$ and $A$ into $A \times_{\alpha, u} K$, respectively.

Let now $\pi$ be a nondegenerate representation of $A$ on some Hilbert space $\mathcal{H}=\mathcal{H}_{\pi}$ and let $\pi_{\alpha}$ be the associated representation of $A$ on $\mathcal{H}_{K}=\ell^{2}(K, \mathcal{H})$ defined by

$$
\left(\pi_{\alpha}(a) \xi\right)(k)=\pi\left(\alpha_{k^{-1}}(a)\right) \xi(k), a \in A, \xi \in \mathcal{H}_{K}, k \in K .
$$

For every $k \in K$, let $\lambda_{u}(k)$ be the unitary operator on $\mathcal{H}_{K}$ given by

$$
\left(\lambda_{u}(k) \xi\right)(l)=\pi\left(u\left(l^{-1}, k\right)\right) \xi\left(k^{-1} l\right), k, l \in K, \xi \in \mathcal{H}_{K} .
$$

(Note that we follow [22] here; the right-hand version is used in [16].)

The pair $\left(\pi_{\alpha}, \lambda_{u}\right)$ is then a covariant representation of $(A, K, \alpha, u)$; that is,

$$
\begin{aligned}
\pi_{\alpha}\left(\alpha_{k}(a)\right) & =\operatorname{Ad}\left(\lambda_{u}(k)\right)\left(\pi_{\alpha}(a)\right), & k \in K, a \in A ; \\
\lambda_{u}(k) \lambda_{u}(l) & =\pi_{\alpha}(u(k, l)) \lambda_{u}(k l), & k, l \in K .
\end{aligned}
$$

This covariant representation induces a nondegenerate representation Ind $\pi$ of $A \times_{\alpha, u} K$ on $\mathcal{H}_{K}$ determined by

$$
(\operatorname{Ind} \pi)(f)=\sum_{k \in K} \pi_{\alpha}(f(k)) \lambda_{u}(k), \quad f \in \ell^{1}(K, A),
$$

that is, by

$$
(\operatorname{Ind} \pi)\left(i_{A}(a)\right)=\pi_{\alpha}(a),(\operatorname{Ind} \pi)\left(i_{K}(k)\right)=\lambda_{u}(k), a \in A, k \in K .
$$

For each $k \in K$, let $\mathcal{H}_{k}$ denote the copy of $\mathcal{H}$ in $\mathcal{H}_{K}$ given by

$$
\mathcal{H}_{k}=\left\{\xi \in \mathcal{H}_{K} \mid \xi(l)=0 \text { for all } l \in K, l \neq k\right\},
$$

giving us the natural direct sum decomposition $\mathcal{H}_{K}=\bigoplus_{k \in K} \mathcal{H}_{k}$.

Assume now that $\pi^{\prime}$ is a nondegenerate representation of $A$ on $\mathcal{H}^{\prime}$ and denote by $\left(\pi_{\alpha}^{\prime}, \lambda_{u}^{\prime}\right)$ the associated covariant representation of $(A, K, \alpha, u)$ on $\mathcal{H}_{K}^{\prime}$.

Let $T \in \mathcal{B}\left(\mathcal{H}_{K}, \mathcal{H}_{K}^{\prime}\right)$. Denote by $\left[T_{k, l}\right]_{k, l \in K}$ the matrix of $T$ with respect to the natural direct sum decompositions of $\mathcal{H}_{K}$ and $\mathcal{H}_{K}^{\prime}$, and identify each $T_{k, l}$ as an element in $\mathcal{B}\left(\mathcal{H}, \mathcal{H}^{\prime}\right)$.

Hence, if $\eta \in \mathcal{H}$ and $k, l \in K$, then $T_{k, l} \eta=\left(T \eta_{l}\right)(k)$, where $\eta_{l} \in \mathcal{H}_{K}$ is given by $\eta_{l}(k)=\eta$ when $k=l$, and $\eta_{l}(k)=0$ otherwise.

Some tedious (but straightforward) computations give:

(1) $\left(T \pi_{\alpha}(a)\right)_{k, l}=T_{k, l} \pi\left(\alpha_{l^{-1}}(a)\right), \quad\left(\pi_{\alpha}^{\prime}(a) T\right)_{k, l}=\pi^{\prime}\left(\alpha_{k^{-1}}(a)\right) T_{k, l}$,

(2) $\left(T \lambda_{u}(j)\right)_{k, l}=T_{k, j l} \pi\left(u\left(l^{-1} j^{-1}, j\right)\right),\left(\lambda_{u}^{\prime}(j) T\right)_{k, l}=\pi^{\prime}\left(u\left(k^{-1}, j\right)\right) T_{j-1}, l$. 
Proposition 3.1. Assume that $\pi$ and $\pi^{\prime}$ are irreducible and that $\pi \circ \alpha_{j} \nsucceq \pi^{\prime}$ for all $j \in K, j \neq e$. Let $T \in \mathcal{B}\left(\mathcal{H}_{K}, \mathcal{H}_{K}^{\prime}\right)$ intertwine $\operatorname{Ind} \pi$ and Ind $\pi^{\prime}$. Then $T_{k, k}$ intertwines $\pi$ and $\pi^{\prime}$ for all $k \in K$. Further, $T$ is decomposable; that is, $T_{k, l}=0$ for all $k \neq l$ in $K$.

Proof. We first note that $T \pi_{\alpha}(a)=\pi_{\alpha}^{\prime}(a) T$ for all $a \in A$. Using (1), we then get

(3) $T_{k, l} \pi\left(\alpha_{l^{-1}}(a)\right)=\pi^{\prime}\left(\alpha_{k^{-1}}(a)\right) T_{k, l}$ for all $k, l \in K, a \in A$.

Letting $l=k$, this clearly implies that $T_{k, k}$ intertwines $\pi$ and $\pi^{\prime}$ for all $k \in K$.

Assume now that $k \neq l$. Using (3) with $a=\alpha_{k}(b)$, we get

(4) $T_{k, l}\left(\pi \circ \operatorname{Ad}\left(u\left(l^{-1}, k\right)\right) \circ \alpha_{l^{-1} k}\right)(b)=\left(\pi^{\prime} \circ \operatorname{Ad}\left(u\left(k^{-1}, k\right)\right)\right)(b) T_{k, l}$ for all $b \in A$.

From the assumption, we have $\pi^{\prime} \nsucceq \pi \circ \alpha_{l^{-1} k}$. Hence, it follows that $\pi \circ$ $\operatorname{Ad}\left(u\left(l^{-1}, k\right)\right) \circ \alpha_{l^{-1} k}$ and $\pi^{\prime} \circ \operatorname{Ad}\left(u\left(k^{-1}, k\right)\right)$ are irreducible and inequivalent. But (4) says that $T_{k, l}$ intertwines these two representations of $A$, and we can therefore conclude that $T_{k, l}=0$.

The following corollary is due to Zeller-Meier in the case where $u$ takes values in the center of $A$ (see 22, Propositions 3.8 and 4.4]). Part (a) could be deduced from [19, Theorem], but as we also need part (b), we prove both.

Corollary 3.2. (a) Ind $\pi$ is irreducible whenever $\pi$ is irreducible and the stabilizer subgroup $K_{\pi}=\left\{k \in K \mid \pi \circ \alpha_{k} \simeq \pi\right\}$ is trivial.

(b) Assume that $\pi$ and $\pi^{\prime}$ both are irreducible.

Then Ind $\pi \not$ Ind $\pi^{\prime}$ whenever $\pi \circ \alpha_{j} \nsucceq \pi^{\prime}$ for all $j \in K$.

Proof. (a) Suppose that $\pi$ is irreducible and $K_{\pi}$ is trivial. Let $T \in \mathcal{B}\left(\mathcal{H}_{K}\right)$ lie in the commutant of (Ind $\pi)\left(A \times_{\alpha, u} K\right)$. Using Proposition 3.1 with $\pi^{\prime}=\pi$, it follows that $T$ is decomposable and $T_{k, k} \in \pi(A)^{\prime}$ for all $k \in K$. As $\pi$ is irreducible, this gives that $T_{k, k} \in \mathbb{C} I_{\mathcal{H}}$ for all $k \in K$. Further, we have $T \lambda_{u}(j)=\lambda_{u}(j) T$ for all $j \in K$. Hence, using (2), we get

$$
\begin{gathered}
\pi\left(u\left(k^{-1}, k l^{-1}\right)\right) T_{k, k}=T_{k, k} \pi\left(u\left(k^{-1}, k l^{-1}\right)\right)=\left(T \lambda_{u}\left(k l^{-1}\right)\right)_{k, l} \\
=\left(\lambda_{u}\left(k l^{-1}\right) T\right)_{k, l}=\pi\left(u\left(k^{-1}, k l^{-1}\right)\right) T_{l, l},
\end{gathered}
$$

which implies that $T_{k, k}=T_{l, l}$ for all $k, l \in K$. Altogether, this means that $T$ is a scalar multiple of the identity operator on $\mathcal{H}_{K}$. Hence we have shown that Ind $\pi$ is irreducible, as desired.

(b) Assume that $\pi$ and $\pi^{\prime}$ both are irreducible and $\pi \circ \alpha_{j} \not \pi^{\prime}$ for all $j \in K$. Let $T \in \mathcal{B}\left(\mathcal{H}_{K}, \mathcal{H}_{K}^{\prime}\right)$ intertwine Ind $\pi$ and Ind $\pi^{\prime}$. It follows from Proposition 3.1 that $T_{k, l}=0$ for all $k, l \in K, k \neq l$, and that $T_{k, k}$ intertwine $\pi$ and $\pi^{\prime}$ for all $k \in K$. As $\pi \neq \pi^{\prime}$ by assumption, we also have $T_{k, k}=0$ for all $k \in K$. Hence, $T=0$. This shows that Ind $\pi \neq$ Ind $\pi^{\prime}$, as desired.

Actually, both implications converse to those stated in (a) and (b) of Corollary 3.2 also hold (as in 22]). However, since we do not need these in this paper, we skip the proofs.

\section{REFERENCES}

1. M. Bekka, M. Cowling, P. de la Harpe, Simplicity of the reduced $C^{*}$-algebra of PSL $(n, \mathbb{Z})$. Internat. Math. Res. Notices 1994, no. 7, 285ff., approx. 7 pp. (electronic). MR 1283024 (95h:22005)

2. M. Bekka, M. Cowling, P. de la Harpe, Some groups whose reduced $C^{*}$-algebra is simple, Inst. Hautes Études Sci. Publ. Math. 80 (1994), 117-134. MR1320606 (96a:22020) 
3. B. Bekka, P. de la Harpe, Irreducibly represented groups. Comment. Math. Helv. 83 (2008), 847-868. MR2442965 (2009e:22004)

4. B. Bekka, P. de la Harpe, A. Valette, Kazhdan's Property (T), New Mathematical Monographs, vol. 11, Cambridge University Press, 2008. MR2415834 (2009i:22001)

5. M.D. Choi, The full $C^{*}$-algebra of the free group on two generators. Pac. J. Math. 87 (1980), 41-48. MR.590864 (82b:46069)

6. K.R. Davidson, $C^{*}$-algebras by example, Fields Institute Monographs, 6, Amer. Math. Soc., 1996. MR.1402012(97i:46095)

7. J. Dixmier, Les $C^{*}$-algèbres et leurs représentations, Gauthier-Villars, Paris, 1969. MR 0246136 (39:7442)

8. P. de la Harpe, On simplicity of reduced $C^{*}$-algebras of groups. Bull. Lond. Math. Soc. 39 (2007), 1-26. MR2303514 (2008a:22004)

9. N. Khattou, Deux propriétés de la $C^{*}$-algèbre maximale de certains produits libres. Comptes rendus de la première recontre maroco-andalouse sur les algèbres et leurs applications (Tétouan, 2001), Univ. Abdelmalek Essaâdi, Fac. Sci. Tétouan, Tétouan, 2003, pp. 54-63. MR2029849 (2004i:46084)

10. E. Kirchberg, On nonsemisplit extensions, tensor products and exactness of group $C^{*}$ algebras. Invent. Math. 112 (1993), 449-489. MR.1218321 (94d:46058)

11. G. Mackey, Borel structure in groups and their duals. Trans. Amer. Math. Soc. 85 (1957), 134-165. MR0089999 (19:752b)

12. G. Mackey, Unitary representations of group extensions. I, Acta Math. 99 (1958), 265-311. MR 0098328 (20:4789)

13. G. Mackey, Induced representations and normal subgroups, Proc. Int. Symp. Linear Spaces, Pergamon, Oxford, 1961, pp. 319-326. MR0139687 (25:3118)

14. G.J. Murphy, Primitivity conditions for full group $C^{*}$-algebras. Bull. Lond. Math. Soc. 35 (2003), 697-705. MR1989500 (2004d:46062)

15. J.A. Packer, Twisted group $C^{*}$-algebras corresponding to nilpotent discrete groups. Math. Scand. 64 (1989), 109-122. MR1036431 (91e:46091)

16. J.A. Packer, I. Raeburn, Twisted crossed products of $C^{*}$-algebras. Math. Proc. Camb. Phil. Soc. 106 (1989), 293-311. MR1002543 (90g:46097)

17. G.K. Pedersen, $C^{*}$-algebras and their automorphisms groups, Academic Press, London, 1979. MR548006 (81e:46037)

18. J.C. Quigg, On the irreducibility of an induced representation. I, Pacific J. Math. 93 (1981), 163-179. MR621605 (84j:22007)

19. J.C. Quigg, On the irreducibility of an induced representation. II, Proc. Amer. Math. Soc. 86 (1982), 345-348. MR667304 (84m:22010)

20. J-P. Serre, Trees, Springer-Verlag, Berlin, 2003. MR.1954121 (2003m:20032)

21. H. Yoshizawa, Some remarks on unitary representations of the free group. Osaka Math. J. 3 (1951), 55-63. MR0041854(13:10h)

22. G. Zeller-Meier, Produits croisés d'une $C^{*}$-algèbre par un groupe d'automorphismes. J. Math. Pures Appl. (9) 47 (1968), 101-239. MR0241994 (39:3329)

Institute of Mathematics, University of Oslo, P.O. Box 1053 Blindern, 0316 Oslo, NORWAY

E-mail address: bedos@math.uio.no

Department of Mathematical Sciences, Norwegian University of Science and TechNOLOGY, 7491 TRONDHEIM, NORWAY

E-mail address: tronanen@math.ntnu.no 